addition to the mediator necessary for solution of the individual problems, a common mediator such as "the new one" was utilized by that group.

The difference between the type of learning set for the kindergartners and first graders compares with the distinction made by Reese (1963) between learning sets and performance sets. Performance sets are a generalized learning-to-learn whereas learning sets involve the acquisition of solutions common to a number of problems The present findings that improvement in performance over problems was not influenced by the order of occurrence of the particular problems lends support to the view that the first graders were acquiring a learning set.

The present results suggest that learning sets for simple concepts could provide a useful mechanism for the study of age-related mediating mechanisms. The specific reversal task proved simple for the first graders as evidence by the ceiling effect on RT. The presence of new irrelevant dimensions during the reversal shifts or the use of other intradimensional shifts as described by Wolff (1967) might provide a more sensitive index of age differences.

\section{REFERENCES}

Brown, A. L., \& Scott, M. S. Transfer between oddity and relative size concepts: reversal and extradimensional shifts. Journal of Experimental Child Psychology, 1972, 13, 350-367.

Goss, A. E. Verbal mediating responses and concept formation. Psychological Review, 1961, 68, 248-274.

Kendler, H. H., \& Kendler, $T$. S. Vertical and horizontal processes in problem solving. Psychological Review, 1962, 69, $1-16$.

Kendler, T. S. An ontogeny of mediational deficiency. Child Development, 1972, 43, 1-17.

Kendler, T. S., \& Kendler, H. H. Reversal and nonreversal shifts in kindergarten children. Journal of Experimental Psychology, $1959,58,56-60$.

Kendler, T. S., Kendler, H. H., \& Wells, D. Reversal and nonreversal shifts in nursery school children. Journal of Comparative and Physiological Psychology, 1960, 53, 83-88.

Reese, H. W. Discrimination learning set in children. In L. P. Lipsitt and C. C. Spiker (Eds.)., Advances in child development and behavior, Vol. 1, New York: Academic Press, 1963.

Savaro, A., \& Kolodny, M. Learning set and shift behavior in children. Journal of Experimental Child Psychology, 1969, 7, 21-30.

Wolff, J. L. Concept-shift and discrimination-reversal learning in humans. Psychological Bulletin, 1967, 68, 369-408.

\title{
Memory search as a function of phonological context
}

\author{
ROBERT B. DICK and LARRY HOCHHAUS \\ Oklahoma State University, Stillwater, Oklahoma 74074
}

\begin{abstract}
The 11 subjects memorized variable lists of either one or three spoken consonant-vowel (CV) syllables. Each list (positive set) was followed by a spoken CV test syllable and subject's task was to indicate whether or not the test syllable matched a syllable from the list. Negative test probes were either phonemically distinct from items in the list or were phonemically similar to list items. Reaction time (RT) increased with list length and RT was faster to positive than to negative probes. Additionally, a significantly faster memory scanning rate was observed for phonemically distinct negative probes (53 msec/item) than for negative test probes which were similar to items in the positive set $(81 \mathrm{msec} /$ item $)$. Contrary to earlier research it was concluded that phonological distinctive features have a large effect on memory search RT.
\end{abstract}

Analysis of distinctive features of stimulus displays has occupied several researchers in recent years. Gibson's (1969) recently proposed theory of perceptual learning hypothesized that differentiation in visual displays involves the searching and processing of the distinctive

The research for this paper was supported by Oklahoma State University research funds. This paper is sponsored by Robert F Stanners who takes full editorial responsibility for it.

Robert Dick is now at National Institute of Mental Health, Regional Office IV, Atlanta, Georgia.

Requests for reprints should be addressed to Larry Hochhaus, Department of Psychology, Oklahoma State University, Stillwater, Oklahoma 74074. features present. Employing a disjunctive reaction time task, Yonas and Gibson (1967) found that a visual distinctive feature (diagonality) common to all positive set items produced greater reduction in latencies with practice than was found when the diagonality feature was present in both positive set and negative set items.

The auditory analog and historical basis of Gibson's visual distinctive features is the phonemic distinctive feature. According to Jakobson, Fant, and Halle (1967), any one language code has a finite set of distinctive features and a finite set of rules for grouping these 
features into phonemes. In an auditory Sternberg-type task, Foss and Dowell (1971) failed to replicate the results of Yonas and Gibson; similar positive set phonemes did not decrease memory search reaction time (RT) relative to the condition of dissimilar positive set phonemes.

While presentation mode and task differences might account for the failure to replicate, at least two additional factors may have contributed to Foss and Dowell's negative results. First, regarding experimental materials, similar and dissimilar phonemes were not systematically chosen and, second, the phonemes to be recognized were each presented as the initial phoneme of a word. Perhaps the presence of other phonemes made it difficult for subjects to scan memory on the basis of the initial phoneme's distinctive features.

To accommodate these criticisms, phonemic materials in the present experiment were systematically chosen from Brown's three-dimensional distinctive feature matrix (Brown, 1965). Also, phonemes in the present task were presented as spoken consonant-vowel (CV) syllables; the vowels in each syllable were a constant $[-0]$ sound.

Additionally, to more precisely demonstrate the subject's use of distinctive features in short-term memory search, the conditions of phonemic similarity and dissimilarity were drastically altered in the present task. Instead of manipulating the distinctive feature similarity of positive set items (as did Foss \& Dowell, 1971), the present procedures manipulated the similarity between negative probes and the positive set items (which were homogeneous with respect to intraset similarity). The present design is most like that of Neisser (1964) who, in a visual search task, found that similarity between target and nontarget items had a large effect on the visual scanning rate. For example, Neisser found that the letter $\mathrm{Z}$ was identified faster in the context of letters with rounded lines than in the context of a straight line letters. For the present experiment it was hypothesized that RT is faster for "far" negative probes (those phonemically distinct from positive set syllables) than for "near" negative probes (probes similar to positive set items).

\section{METHOD}

\section{Subjects}

The subjects were 11 Oklahoma State University undergraduates recruited voluntarily from undergraduate psychology sources. Each subject was tested in a single session of 144 trials preceded by 12 practice trials. The single session lasted about $1 \mathrm{~h}$ with a 5 -min break at the halfway point.

\section{Stimulus Materials}

Brown's (1965) distinctive features matrix for eight consonants was used to construct the stimulus lists. The vowel $[-0]$ was added to each consonant, and lists of one and three CV syllables were recorded on tape for presentation to the subjects. Probes were also CV syllables, with the positive probe a member of a previously presented list, or set, and a negative probe not a member of the previously presented set. Negative probes were also chose according to a "near" and "far" distinction based on the features of the matrix. For set size 3 , a "near" negative probe had two features in common with one item in the memory set and one feature in common with the other two items in the memory set. A "far" probe had one feature in commor with two items in the memory set and no features in common with two items in the memory set and no features in common with the third item in the set. In Set Size 1, a "near" probe had two features in common with the single memory item and a "far" probe had no features in common with the single memory item. The CV syllables used were $/ \mathrm{bo} /, / \mathrm{po} /$, $/ \mathrm{vo} /, / \mathrm{to} /, / \mathrm{do} /, / \mathrm{fo} /, \mid \theta \mathrm{o} /$, and $/ \mathrm{\sigma o}_{\mathrm{o}} /$.

\section{Procedure}

Stimulus materials were presented auditorily on a Realistic tape recorder over headphones. The left channel of the stereo recorder was used to record the memory sets and probes for presentation to the subjects. Probes were simultaneously recorded in the right channel and this channel was used to activate a voice key which started a Hunter Klockounter. Simultaneous recording of the probes in both channels resulted in the voice key (Gerbrands) firing the Klockounter at the same time the subject heard the probe stimulus. The Klockounter stopped when the subjects made their response to the probe by moving a two-way toggle switch mounted on a table in front of them to either a yes or a no position. Reaction time in milliseconds was recorded from the Klockounter, and positive and negative responses were counterbalanced with the left and right toggle switch responses. Each trial was initiated by the word "ready," followed $2 \mathrm{sec}$ later by the meory set (one or three consonant-vowel syllables). A 5-sec pause occurred before the probe item was presented. Items in the memory set were spaced at $1-\mathrm{sec}$ intervals, and there was a $10-\mathrm{sec}$ interval between each trial.

Subjects were presented with 144 trials arranged into two trial blocks. There was a 5-min rest period between each block and 12 practice trials preceded the experimental trials. One half of the trials involved Set Size 3 and half were of Set Size 1. There were an equal number of positive and negative probes, and one half of the negative probes were "near" negative and one half were "far" negative. In the positive Set Size 3, the one, two, and three positions were probed eight times each. Each trial consisted of a memory set (one or three items) and a probe (positive or negative). Trials were randomly arranged for presentation to the subjects.

\section{RESULTS}

Mean reaction times were calculated for each subject within each category. No block differences were indicated, so the data were collapsed across blocks for subsequent analysis. A completely within-subjects two-factor ANOVA indicated significant differences in scanning rate between probe type $(\mathrm{F}=12.08 \mathrm{df}=1 / 10$, $\mathrm{p}<.01)$ and set size $(\mathrm{F}=17.16, \mathrm{df}=1 / 10, \mathrm{p}<.01)$. Significant interactions occurred between Subjects by Probe Type $(\mathrm{F}=5.01, \mathrm{df}=1 / 10, \mathrm{p}<.01)$. There was no significant interaction between probe type and set size, indicating minimal slope differences. Regression analysis on the same data indicated a scanning rate of about $71 \mathrm{msec}$ per item using the positive probe and $67 \mathrm{msec}$ per item with the negative probe.

Further analysis of the negative probe items revealed 
Table 1

Mean Reaction Time and Error Rates for Each Condition Over All Trials

\begin{tabular}{|c|c|c|c|c|c|c|c|c|}
\hline \multirow{3}{*}{ Probe Type } & \multicolumn{4}{|c|}{ Set Size 1} & \multicolumn{4}{|c|}{ Set Size 3} \\
\hline & \multirow[t]{2}{*}{ Positive } & \multicolumn{3}{|c|}{ Negative } & \multirow[t]{2}{*}{ Positive } & \multicolumn{3}{|c|}{ Negative } \\
\hline & & Combined & Near & Far & & Combined & Near & Far \\
\hline Mean RT & .521 & .596 & 619 & .592 & .806 & .865 & .941 & .805 \\
\hline Number of Errors & 21 & 7 & 6 & 1 & 58 & 44 & 36 & \\
\hline Percent Error & 5.3 & 1.8 & 3.0 & .5 & 14.6 & 11.1 & 18.2 & 4.0 \\
\hline
\end{tabular}

the most interesting results. With no block differences, scores on negative probes were totaled and averaged over all trials according to set size and the near-far classification giving a mean reaction time for each subject. A matched-groups $t$ test supported the distinctive features hypothesis in Set Size 3 showing greater search times for the near negative probes $(t=$ $1.31, \mathrm{df}=10, \mathrm{p}<.01)$. Set Size 1 differences were not significarit $(t=1.31, \mathrm{df}=10, \mathrm{p}<.30)$. The least-squares equation on the same data revealed a scanning rate of $81 \mathrm{msec}$ per item for the near negative probe and $53 \mathrm{msec}$ per item for the far negative probes (See Figure 1).

A second analysis of variance was performed on the subjects' mean RTs to the positive probe (serial position and subjects were the two factors) and revealed a significant serial position effect, with the faster RTs occurring in positions one and three and slower RTs in position two $(\mathrm{F}=4.14, \mathrm{df}=2 / 18, \mathrm{p}<.05)$. A Newman-Keuls test further substantiated the significance of both a primacy and a recency effect in the serial position data. Table 1 presents a summary of the error data and mean reaction times for all subjects in each condition over all trials. Examination of the error tabulations reveals the highest error rates occurred in set size three with the positive and negative probes.

\section{DISCUSSION}

The results clearly indicate the importance of stimulus context in memory scanning. Items which are more phonetically distinct from other items are more quickly identified. This view is supported by the significant difference obtained in Set Size 3, where the far probe (maximum of one distinctive feature overlap) produced faster reaction time than the near probe (two distinctive features overlap). Failure to obtain significance in Set Size 1 could probably be attributed to size of the memory set, which may have limited the advantage of using distinctive features. With more distinctive feature overlap, there is more confusability in processing the items. However, when only one item is being scanned the effect is limited.

Overall, the scanning rates for the positive and negative probes were approximately equal ( 71 and $67 \mathrm{msec} / \mathrm{item})$, but when the negative probe was analyzed according to the underlying distinctive features, marked differences occur. The near negative probes $(81 \mathrm{msec} /$ item $)$ are slower than the far negative probes $(53 \mathrm{msec} /$ item $)$. The data clearly indicate that the short-term memory scanning rate can be affected by the stimulus context.

These results do differ from the previous study by Foss and Dowell (1971) which used phonemic distinctive features in a
Sternberg (1969) type task. The main differences between the studies were referred to in the introduction and included use in the present study of consonant-vowel similarity to the negative set instead of the positive set, and the use of Brown's distinctive features matrix which appears more concise than the system used in the Foss and Dowell study. Additionally examination of the subject's error data reveals a possible explanation for the discrepancy in results (see Table 1). The subjects in this experiment had their highest error rates in Set Size 3 with the positive and near negative probes $(14.6 \%$ and $18.2 \%)$ indicating the level of acoustic confusability was very high. It could be that with too much distinctive feature overlap, the level of acoustic confusability is so high that subjects perform close to chance on some items. In most cases, operating at the chance level on these items should increase the scanning rate for the items. The reduced scanning rate and error rates with the far negative probes, were the distinctive features overlap is minimal, seems to support this notion. Previous research has shown error rates affected by acoustic confusability in short-term memory (Wicklegren, 1965).

The results in the present study support a recent study by Lyons and Briggs (1971) who manipulated the context of the stimulus material presented and accuracy of response. They found the intercept varied with the accuracy levels while the slopes varied with the stimulus material. Examination of Figure 1 of the present report shows the relative closeness of the intercepts for the negative response, but a subsequent divergence as the material is scanned (the difference between scanning one and three items.).

\section{REFERENCES}

Brown, R. Social psychology. New York: Free Press, 1965.

Burrows, D., \& Okada, R. Serial position effects in high-speed memory search. Perception \& Psychophysics, 1971, 10, 305-308.

Foss, D. J., \& Dow:ll, B. E. High-speed memory retrieval with auditorily presented stimuli. Perception \& Psychophysics, $1971,9,465-468$.

Gibson, E. J. Principles of perceptual learning and development. New York: Appleton-Century-Crofts, 1969.

Jakobson, R., Fant, C. G. M., \& Halle, M. Preliminaries to speech analysis: The distinctive features and their correlates. Cambridge, Mass: The M.I.T. Press, 1967.

Lyons, J. J., \& Briggs, G. E. Speed-accuracy trade-off with different types of stimuli. Journal of Experimental Psychology, 1971, 91, 115-119.

Neisser, U. Visual search. Scientific American, 1964, 210, 94-102.

Sternberg, S. Memory-scanning: Mental processes revealed by reaction-time experiments. American Scientist, 1969, 57, 421-457.

Yonas, A. \& Gibson, E. J. A developmental study of feature processing strategies in letter discrimination. Paper presented at the meeting of the Eastern Psychological Association, Boston, April 1967.

Wickelgren, W. A. Distrinctive features and errors in short-term memory for English words. Journal of the Acoustical Society of America, 1965, 38, 583-588.

(Received for publication November 13, 1974.) 